\title{
Enzyme Replacement Therapy for Murine Mucopolysaccharidosis Type VII Leads to Improvements in Behavior and Auditory Function
}

\author{
Lynn H. O’Connor, ${ }^{\star}$ Lawrence C. Erway, Carole A. Vogler, ${ }^{ף}$ William S. Sly, ${ }^{\star \star}$ Andrew Nicholes, ${ }^{\ddagger}$ Jeff Grubb, ${ }^{\star \star}$ \\ Sandra W. Holmberg, ${ }^{*}$ Beth Levy, ${ }^{\mathbb{I}}$ and Mark S. Sands ${ }^{\ddagger \S}$ \\ $*$ Department of Psychiatry, ${ }^{\ddagger}$ Department of Internal Medicine, and ${ }^{\S}$ Department of Genetics, Washington University School of Medicine, \\ St. Louis, Missouri 63110; "Department of Biological Sciences, University of Cincinnati, Cincinnati, Ohio 45221; " Department of \\ Pathology, and **The Edward A. Doisy Department of Biochemistry and Molecular Biology, St. Louis University School of Medicine, \\ St. Louis, Missouri 63104
}

\begin{abstract}
Mucopolysaccharidosis type VII (MPS VII; Sly syndrome) is one of a group of lysosomal storage diseases that share many clinical features, including mental retardation and hearing loss. Lysosomal storage in neurons of the brain and the associated behavioral abnormalities characteristic of a murine model of MPS VII have not been shown to be corrected by either bone marrow transplantation or gene therapy. However, intravenous injections of recombinant $\beta$-glucuronidase initiated at birth reduce the pathological evidence of disease in MPS VII mice. In this study we present evidence that enzyme replacement initiated at birth improved the behavioral performance and reduced hearing loss in MPS VII mice. Enzyme-treated MPS VII mice performed similarly to normal mice and significantly better than mocktreated MPS VII mice in every phase of the Morris Water Maze test. In addition, the auditory function of treated MPS VII mice was dramatically improved, and was indistinguishable from normal mice. These data indicate that some of the learning, memory, and hearing deficits can be prevented in MPS VII mice if enzyme replacement therapy is initiated early in life. These data also provide functional correlates to the biochemical and histopathological improvements observed after enzyme replacement therapy. $(J$. Clin. Invest. 1998. 101:1394-1400.) Key words: lysosomal storage diseases - central nervous system • mental retardation • behavioral sciences $\cdot$ auditory evoked response
\end{abstract}

\section{Introduction}

Lysosomal storage diseases are inherited disorders usually caused by a deficiency in a single acid hydrolase (1). Mucopolysaccharidosis type VII (MPS VII $)^{1}$ is a lysosomal storage

Address correspondence to Mark S. Sands, Ph.D., Washington University School of Medicine, Department of Internal Medicine, 660 South Euclid Avenue, Box 8007, St. Louis, MO 63110. Phone: 314362-5494; FAX: 314-362-9333; E-mail: msands@imgate.wustl.edu

1. Abbreviations used in this paper: $\mathrm{ABR}$, auditory-evoked brain stem responses; BMT, bone marrow transplantation; CNS, central nervous system; dB, decibels; MPS VII, mucopolysaccharidosis type VII; kHz, kiloHertz.

J. Clin. Invest.

(C) The American Society for Clinical Investigation, Inc. 0021-9738/98/04/1394/07 \$2.00

Volume 101, Number 7, April 1998, 1394-1400

http://www.jci.org disease caused by a deficiency in $\beta$-glucuronidase activity (2). MPS VII is characterized by a variety of systemic effects such as progressive intralysosomal storage, skeletal deformities, hearing defects, mental impairment, and shortened life span (2). A murine model of MPS VII exists that has a single base pair deletion in the $\beta$-glucuronidase gene, lacks any detectable $\beta$-glucuronidase activity, and shares most of the clinical, biochemical and histopathological features of the human disease (3-5). In addition to the biochemical and histopathological abnormalities, MPS VII mice have significant defects in behavior, as measured by the Morris Water Maze test, and have profound hearing loss (6-8).

The MPS VII mouse has been a particularly useful model with which to study the pathophysiology of the disease, and to develop novel therapeutic approaches for this and other lysosomal storage diseases. Varying degrees of improvement have been achieved in the MPS VII mouse after bone marrow transplantation (BMT), neuronal progenitor cell transplantation, and somatic cell gene replacement therapies (8-16). Some of these therapies resulted in dramatic reductions of lysosomal storage, improvements in skeletal development, and increased life span. However, no improvement in higher mental functions was documented after any of these forms of therapy. Effective treatment of the central nervous system (CNS) in patients with lysosomal storage diseases remains a major challenge.

Recombinant enzyme replacement therapy is an effective means of treating murine MPS VII. Relatively high levels of enzyme activity were detected in many tissues, including the brain, in newborn MPS VII mice $1 \mathrm{~h}$ after an intravenous injection of $\beta$-glucuronidase (17). Lysosomal storage material was reduced or eliminated in many tissues, including some neurons of the CNS, after six weekly injections of enzyme initiated at birth (18). This reduction in lysosomal storage material in neurons of the brain persisted for at least $1 \mathrm{yr}$ after early enzyme replacement (19). A persistent reduction in lysosomal storage material in neurons of the CNS was also achieved with either repeated enzyme administration or a combination of early enzyme replacement followed by BMT (20). Although the MPS VII mice treated with recombinant enzyme had reduced lysosomal storage material in many tissues, it was not clear whether improved histopathology correlated with improved function.

Here we show that MPS VII mice receiving weekly intravenous injections of recombinant $\beta$-glucuronidase initiated at birth have a less severe phenotype and show improvements in the histopathology in the brain and ear. We also show that treated MPS VII mice perform significantly better than untreated MPS VII mice in a spatially oriented learning test. Finally, the hearing deficits observed in untreated MPS VII mice are dramatically reduced after early enzyme replacement ther- 
apy. These data provide functional correlates to the improved histopathological findings in the brain and ear after enzyme replacement therapy. They also suggest that early initiation of enzyme replacement therapy may prevent or delay some of the progressive mental impairment and hearing loss associated with this lysosomal storage disease.

\section{Methods}

MPS VII mice and enzyme replacement. Homozygous mutant (MPS VII, $\left.g u s^{m p s} / g u s^{m p s}\right)$ and phenotypically normal $(+/+$ and $+/ m p s)$ mice were obtained from the B6.C- $H-2^{\mathrm{bm} 1} / \mathrm{ByBir}-g u s^{m p s} /+$ colony maintained by M.S. Sands at the Washington University School of Medicine. Heterozygous mice are completely normal by all criteria tested, except for a $50 \%$ decrease in $\beta$-glucuronidase activity. Recombinant murine $\beta$-glucuronidase was overexpressed in mouse L cells lacking the mannose-6-phosphate receptor, and was purified by standard methods as previously described.

All the mice used in this study were males, and were identified at birth as normals or mutants by the presence or absence of $\beta$-glucuronidase activity in a sample of tissue obtained by toe clipping (10). 12 MPS VII and 11 normal mice were injected through the superficial temporal vein with $28,000 \mathrm{U}$ of $\beta$-glucuronidase activity in $100 \mu \mathrm{l}$ of enzyme dilution buffer $(150 \mathrm{mM} \mathrm{NaCl}, 10 \mathrm{mM}$ Tris [pH 7.5], $1 \mathrm{mM}$ $\beta$-glycerophosphate; 21$)$. The mice received six weekly injections starting on the day they were born. Ten each of normal and MPS VII mice were injected on the same schedule with enzyme dilution buffer alone. All of the mice were treated prophylactically with sulfamethoxazole $(0.8 \mathrm{mg} / \mathrm{ml})$ and trimethoprim $(0.16 \mathrm{mg} / \mathrm{ml})$ in their water for the duration of the Morris Water Maze test.

Morris Water Maze test. All of the mice were acclimated to the pool for a single day $1 \mathrm{wk}$ after their final injection. The acquisition, probe, relearning, and relocation phases of the test were performed as described previously $(6,7)$. In brief, a $10-\mathrm{cm}$ transparent platform was placed $1 \mathrm{~cm}$ beneath the surface of the water and $40 \mathrm{~cm}$ from the wall in the SW quadrant of the pool. The mice were released into the water facing the wall. The time required for the mice to find the platform (latency) was recorded for each trial. Mice were given four trials per day at 180 -s intervals. Each cardinal point was selected at random and used once as a starting point each day. $90 \mathrm{~s}$ were allowed for the mice to find the platform during the trials. If the mice did not find the platform, they were guided to the platform and allowed to stay there for $10 \mathrm{~s}$. The acquisition phase of the test was performed over the first five consecutive days. The probe phase was performed on the day immediately after the acquisition phase. The relearning and relocation phases were performed on days $7-10$ and $11-12$, respectively. The mice were followed with a video tracking system using chromotracking software version 4.02 (San Diego Instruments, San Diego, CA) to assess their behavior. The data was analyzed for statistical significance using ANOVA followed by Fisher's LSD test (protected $t$ test).

Auditory-evoked brain stem responses $(A B R)$. The same mice that were analyzed in the water maze were also tested for hearing by means of the auditory evoked brain stem response as previously described (8). The mice were anesthetized by intraperitoneal injection of $0.2-0.5 \mathrm{ml}$ of tribromoethanol $(20 \mathrm{mg} / \mathrm{ml})$. Needle electrodes were placed subdermally on the top of the head (active), below the left ear (reference), and on the dorsosacrum (ground). Headphones adapted to fit a mouse delivered broadband clicks $(0 \pm 16$ kiloHertz $[\mathrm{kHz}])$ or pure-tone pips $(8,16$ or $32 \mathrm{kHz})$ generated from an Intelligent Hearing System averager (Intelligent Hearing Systems, North Miami, FL). The ABR threshold was determined for each stimulus in descending intensities (10 decibels $[\mathrm{dB}])$, and finally at $\pm 5 \mathrm{~dB}$. Each determined threshold exhibited at least two of the four or five peaks of the ABR waveform within 1-5 ms after the stimulus. ABR thresholds of 40-80 $\mathrm{dB}$ indicate a moderate to severe hearing loss, whereas thresholds above $80 \mathrm{~dB}$ indicate a profound hearing loss. Statistical significance of the hearing results was determined using Student's $t$ test.
Histopathological and biochemical analysis. At $12 \mathrm{wk}$ of age, the mice in all four groups were killed and analyzed biochemically and histologically as previously described $(5,9)$. Samples of brain were collected and immediately immersed in cold $2 \%$ glutaraldehyde and $4 \%$ paraformaldehyde in PBS. The brain tissue sections were embedded in Spurr's resin, and $0.5-\mu \mathrm{m}$ sections of the parietal neocortex, hippocampus, and cerebellum, including meninges, were stained with toluidine blue for evaluation of lysosomal storage. Multiple coronal sections of the brain sectioned in paraffin were also evaluated for histologic abnormalities. The skull was placed in $10 \%$ neutral buffered formalin. The ears were manually dissected and decalcified for several days in $10 \%$ formalin and $40 \mathrm{mM}$ EDTA, and subsequently embedded in paraffin. $8-\mu \mathrm{m}$-thick transverse sections of the external and middle ear were stained with hematoxylin-eosin.

Lysosomal enzyme activities were quantified in homogenates of liver, spleen, and brain as previously described (22). $\beta$-glucuronidase, $\alpha$-galactosidase, and $\beta$-hexosaminidase activities were determined using the fluorescent substrates 4-methylumbelliferyl- $\beta$-D-glucuronide, 4-methylumbelliferyl- $\alpha$-D-galactoside, and 4-methylumbelliferyl- $\beta$-Dglucosaminide, respectively (Sigma Chemical Co., St. Louis, MO).

\section{Results}

Two groups of at least 10 MPS VII mice and 10 normal litter mates received six weekly intravenous injections of $28,000 \mathrm{U}$ of recombinant murine $\beta$-glucuronidase starting on the day they were born. One control group each of MPS VII and normal mice were injected at the same intervals with enzyme dilution buffer. Mice injected with enzyme will be referred to as treated normals or treated MPS VII mice, and mice injected with enzyme dilution buffer will be referred to as untreated normals or untreated MPS VII mice. The MPS VII phenotype, characterized by dwarfism and flattened facial profile, did not develop to the same extent in treated MPS VII mice as that seen in untreated MPS VII mice. The more normal phenotype was still apparent at the end of the study when the mice were $\sim 12$ wk of age (Fig. 1 ).

At 6 wk of age (1 wk after the last injection), all of the mice were acclimated to the pool before the beginning of the acqui-

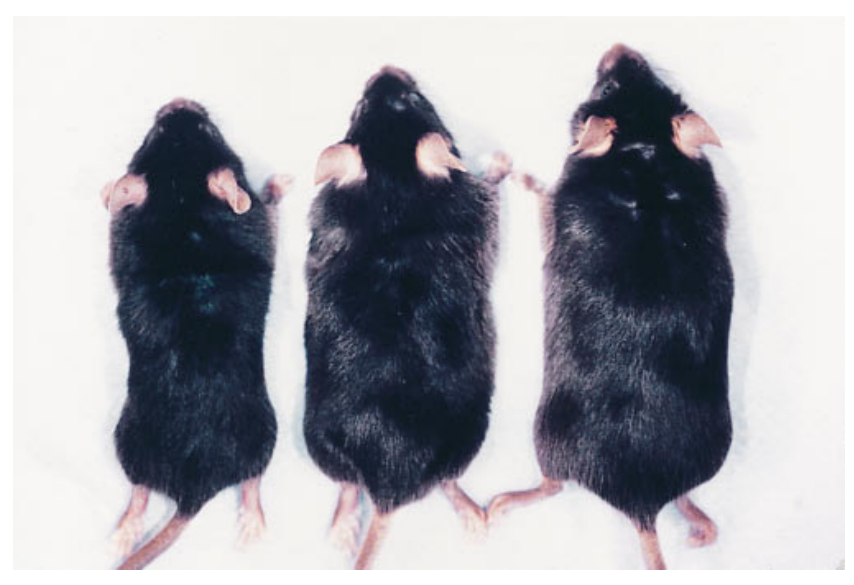

Figure 1. Phenotypic improvements are observed in a treated MPS VII mouse (middle) when compared with an age-matched untreated MPS VII mouse (left). The treated MPS VII mouse is larger, the bone lengths are greater (18-20), and the facial dysmorphism is less severe than in the untreated MPS VII mouse. An age-matched normal mouse that received weekly injections of buffer is shown for comparison (right). 


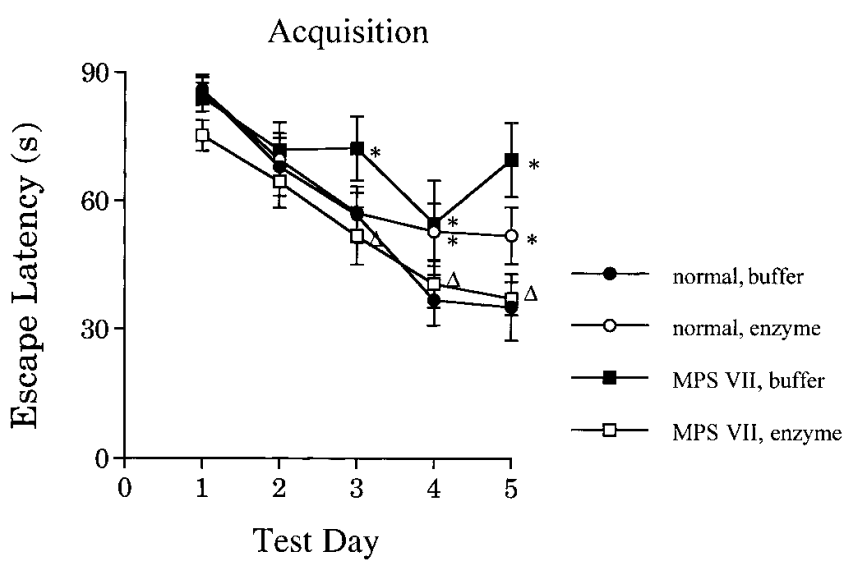

Figure 2. The latencies to find the platform during the acquisition phase of the Morris Water Maze test (days 1-5) of untreated normal $(\bullet)$ and MPS VII ( $\mathbf{\square})$ mice, and treated normal $(\bigcirc)$ and MPS VII $(\square)$ mice are shown. *Significant $(P<0.05)$ difference in the latency when compared with the group of untreated normal mice. $\triangle$, Significant $(P<0.05)$ difference in latency between treated MPS VII mice and untreated MPS VII mice.

sition phase of the Morris Water Maze test (23). There was no significant difference between the untreated normal mice and the treated MPS VII mice throughout the entire acquisition phase of the test (Fig. 2). During the first $2 \mathrm{~d}$ of the acquisition phase, there was no significant difference between the times required to find the platform (latency) in any of the four groups of animals. However, on the third day of testing, the latency to find the platform was significantly longer in the group of untreated MPS VII mice when compared with any of the other three groups. On the fourth and fifth days there was a significant decrease in the latency of both the untreated normal and treated MPS VII groups when compared with either the untreated MPS VII or the treated normal mice.

The probe phase was performed on the sixth day of testing, immediately after the acquisition phase. There was no significant difference in the latencies between the treated MPS VII mice and the untreated normal mice (Fig. $3 A$ ). The latency to cross the region where the platform was previously located was significantly shorter in the group of treated MPS VII mice when compared with the group of untreated MPS VII mice. Treated normal mice had a significantly increased latency compared with both the untreated normals and the treated MPS VII mice. The number of mice that actually crossed over the platform was dramatically different between the various groups. 9 out of 10 and 8 out of 11 animals from the untreated normal and the treated MPS VII groups, respectively, crossed the platform (Fig. $3 A$ ). However, only 3 out of 8 and 2 out of 11 mice from the untreated MPS VII and the treated normal groups, respectively, crossed the platform.

In addition to the differences in latencies to cross the platform during the probe phase, there were also significant differences between the groups with respect to the amount of time the mice spent in the target quadrant searching for the platform (Fig. $3 B$ ). Untreated normal mice and treated MPS VII mice spent significantly more time in the target quadrant compared with the other three quadrants. In the remaining two groups, the time spent in the target quadrant was not significantly greater than the time spent in the other quadrants.

During the relearning phase of the test, the platform was placed in the original position, and the latency to find the platform was measured. There was no significant difference in the latencies between the treated MPS VII mice and the untreated normals (Fig. 4). Untreated MPS VII mice had the longest latency, which was significantly greater than the latencies of any of the other three groups. There was also a significant increase in the latency of the treated normal mice when compared with untreated normal animals.

In the relocation phase of the Morris Water Maze test, the platform was placed in the opposite quadrant relative to the

A

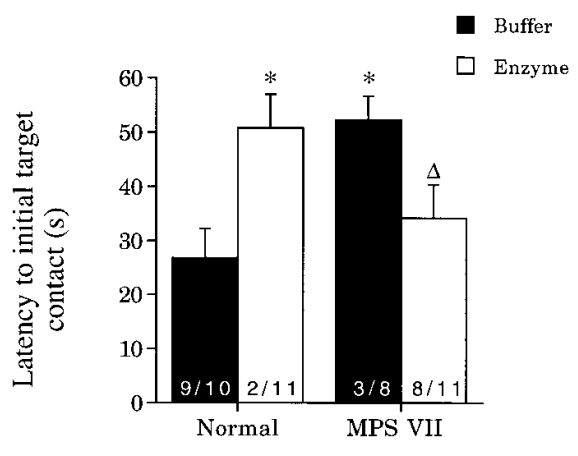

B

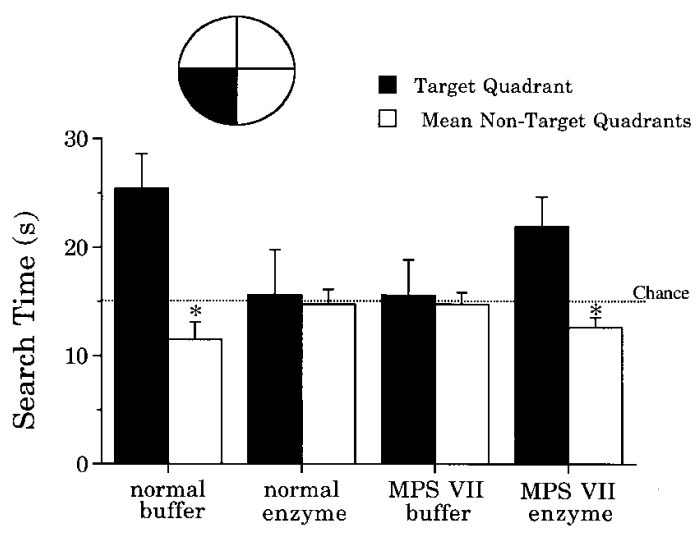

Figure 3. The probe test was performed on day six immediately after the acquisition phase. The latencies of the four groups to cross the area where the platform was previously located are shown $(A)$. Untreated normal and MPS VII mice are indicated by filled bars, and treated normal and MPS VII mice are indicated by open bars. *Significant $(P<0.05)$ increase in the latencies compared with untreated normal mice. $\triangle$, Significant $(P<0.05)$ difference in the latencies between the treated and untreated MPS VII mice. The fractions within the respective bars indicate the number of mice that crossed over the platform (numerator) compared with the total number of mice in that group (denominator). The time(s) the mice spent in the target quadrant searching for the platform is shown $(B)$. Filled bars indicate the time spent in the target quadrant (filled quadrant in circle), and open bars indicate the average time spent in each of the remaining three quadrants (open quadrants). The dotted line (chance) represents the time (15 s) spent in the respective quadrants if the mice swam randomly, i.e., equal amount of time in each quadrant. *Significant $(P<$ 0.05 ) increase in the time spent in the target quadrant compared with the other three quadrants. Two untreated MPS VII mice died before the probe test. 


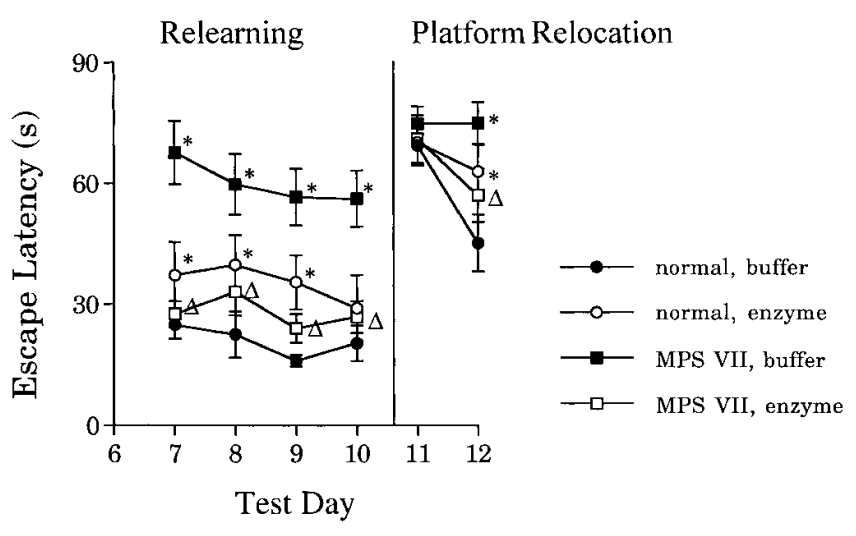

Figure 4. The latencies of the four groups (open squares, treated MPS VII; filled squares, untreated MPS VII; open circles, treated nor$\mathrm{mal}$; filled circles, untreated normal) to find the platform placed in the original quadrant (relearning) and the latencies to find the platform placed in a new position (relocation) are shown. *Significant $(P<$ $0.05)$ increase in latency compared with untreated normal mice. $\triangle$, Significant $(P<0.05)$ difference between treated and untreated MPS VII mice.

original, and the latency to find the platform was measured. There was no significant difference between any of the four groups on the first day of relocation (Fig. 4). The latencies of treated MPS VII mice and untreated normal mice did not differ significantly on the second day. However, the latencies of the two remaining groups were significantly greater than either the treated MPS VII mice or the untreated normal mice.

At $\sim 12$ wk of age, the mice in all four groups were killed, and various tissues including the brain were examined biochemically and histologically. The levels of $\beta$-glucuronidase and the histopathological findings in tissues of treated MPS VII mice were consistent with a previous study (19). $\beta$-glucuronidase activity was undetectable in the brains of treated MPS VII mice, and there was little or no reduction in the secondary elevations of other lysosomal enzymes (data not shown). However, lysosomal storage material was reduced or absent in neocortical neurons in treated MPS VII mice when compared with untreated MPS VII mice (Fig. 5). There were no obvious morphologic abnormalities observed in the brains of treated and untreated normal mice.

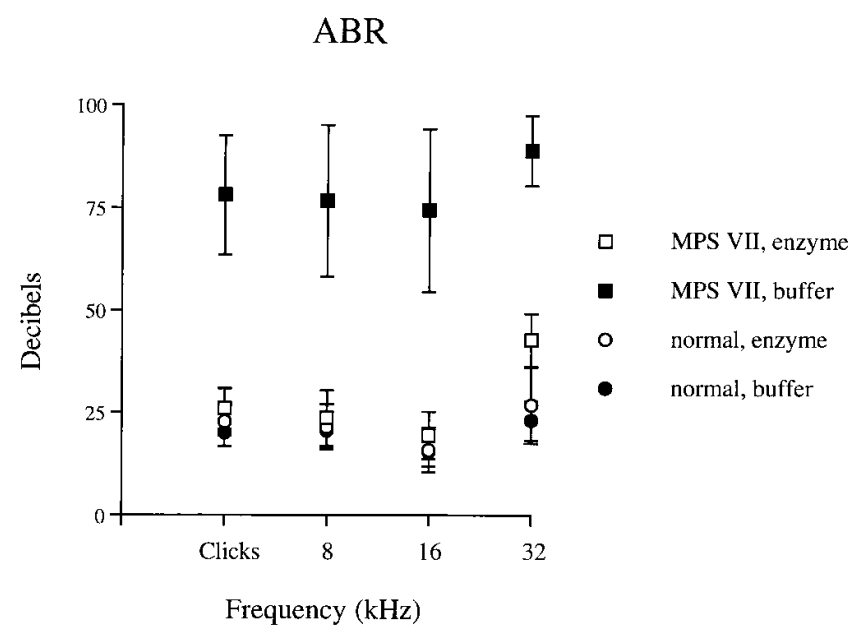

Figure 6. Improvements in auditory function are observed in MPS VII mice after enzyme replacement therapy initiated at birth. There is a significant $(P<0.001)$ decrease in the decibels required to elicit an ABR in the treated MPS VII mice ( $\square$ ) compared with untreated MPS VII mice ( $\square)$. There are no significant differences in the ABRs at the broadband (clicks), 8 and $16-\mathrm{kHz}$ frequencies between the treated MPS VII mice and either the treated $(O)$ or untreated normal (O) mice. There is an increase in the ABR threshold of treated MPS VII mice at $32 \mathrm{kHz}$ compared with the normal mice.

Mice with MPS VII have severe histopathologic abnormalities in the ear that are associated with a profound hearing loss $(8,24)$. Measurements of the ABR have been useful in assessing functional improvements after syngeneic bone marrow transplantation in MPS VII mice (8). The decibels required to elicit an ABR at broadband $(0-\geq 16 \mathrm{kHz}$ ) or specific ( 8 and $16 \mathrm{kHz}$ ) frequencies were indistinguishable among the treated MPS VII mice, untreated normal mice, and treated normal mice (Fig. 6). At $32 \mathrm{kHz}$, treated MPS VII mice had increased thresholds compared with both groups of normal mice, but had ABR thresholds that were lower than those of untreated MPS VII mice. Untreated MPS VII mice required a stimulus $>60$ $\mathrm{dB}$ at every frequency to elicit the characteristic ABR waveform. There was a corresponding improvement in the histopathology of the ear in treated MPS VII mice, although the extent of improvement varied between individual mice, and
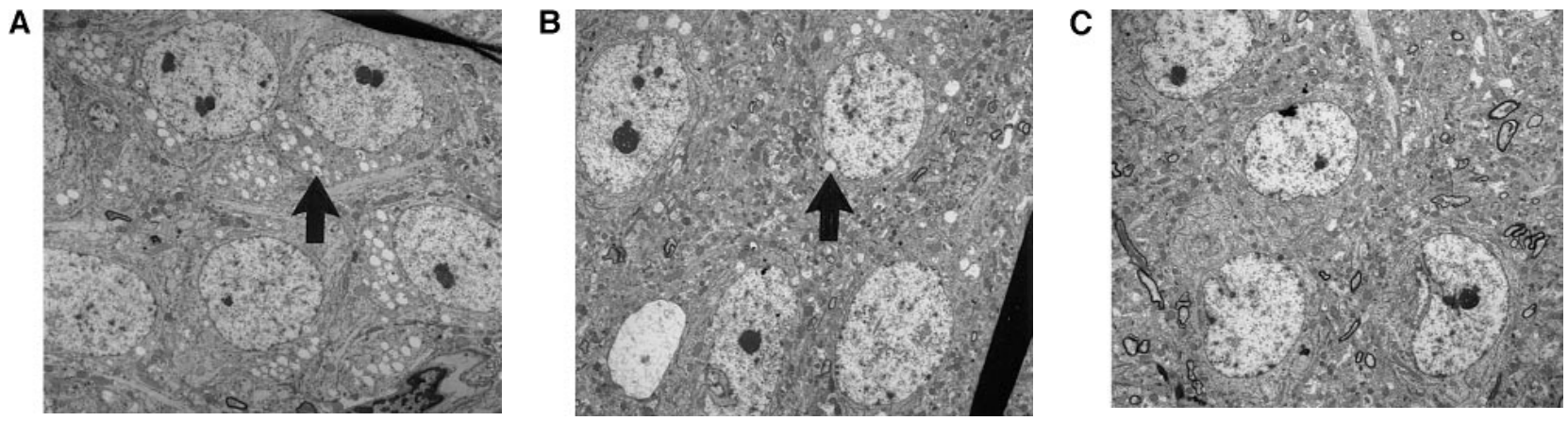

Figure 5. Histopathologic changes in neurons of the cerebral cortex after intravenous injections of $\beta$-glucuronidase or buffer. Lysosomal distension (filled arrows) is observed in neurons from an untreated MPS VII mouse $(A)$. The lysosomal distension (filled arrows) is greatly reduced in neurons from a similar region of the brain in a treated MPS VII mouse $(B)$. There is no evidence of lysosomal storage material in neurons from a treated normal animal $(C)$. $(A-C, 1400 \times$; uranyl acetate-lead citrate). 
A
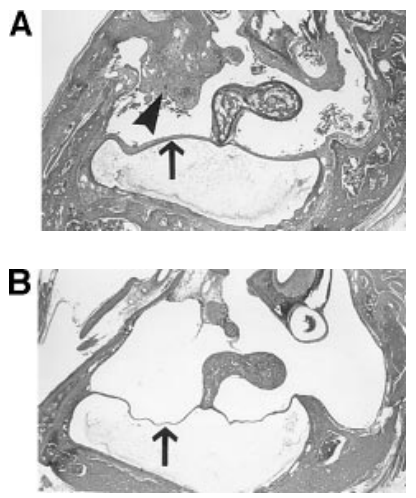

C

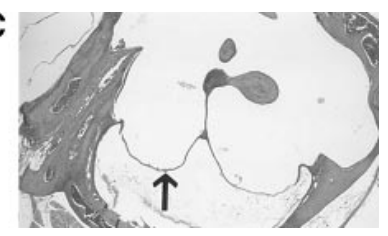

D

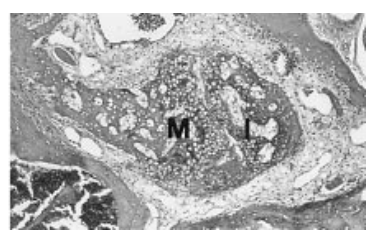

E

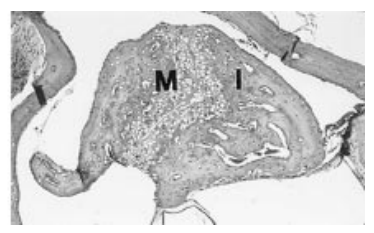

$\mathbf{F}$

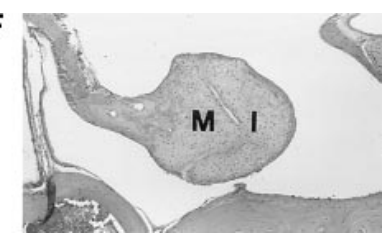

Figure 7. Histopathologic improvements are observed in the ears of treated MPS VII mice. An ear from an untreated MPS VII mouse (A) shows a thickened tympanic membrane (arrow) and inflammation and thickening mucosa in the middle ear (arrowhead). The ossicle attached to the tympanic membrane has expansion of the perivascular spaces due to the lysosomal storage disease. An ear from a treated MPS VII mouse $(B)$ has a thinner tympanic membrane (arrow), less histologic evidence of lysosomal storage, and no otitis. The ossicle, although not normal, shows less histologic evidence of lysosomal storage. An ear from an untreated normal mouse $(C)$ has a thin tympanic membrane (arrow) and no otitis. The malleus $(M)$ and incus $(I)$ from an untreated MPS VII mouse $(D)$ is encased in a thickened mucosa. Articular chondrocytes are distended, and perivascular lacunae are expanded. The malleus and incus from a treated MPS VII mouse $(E)$ show similar articular disease, but less expansion of the perivascular lacunae. The ossicles are not entrapped in mucosa. The malleus and incus from an untreated normal mouse $(F)$ are shown. ( $A-F$, hematoxylin-eosin; $A-C, 16 \times ; D-F, 39 \times)$.

lysosomal storage was not completely eliminated. The malformations of the ossicles were reduced, there was less middle ear inflammation, and the tympanic membranes were thinner in treated MPS VII mice when compared with the untreated MPS VII mice (Fig. 7).

\section{Discussion}

MPS VII is a progressive lysosomal storage disease with multiple clinical symptoms and a characteristic phenotype partially caused by abnormal skeletal development. These data show that injections of recombinant $\beta$-glucuronidase initiated at birth reduce the severity of the phenotype in MPS VII mice. The improvement in clinical appearance is probably due to the increased bone length and body weight (18-20). Similar improvements in clinical appearance were also observed after syngeneic BMT, but only if the BMT was performed at birth (10).

In addition to the phenotypic improvements, MPS VII mice treated with enzyme from birth had histologic evidence of reduced lysosomal storage in many tissues, including neurons of the brain. The reduced lysosomal storage in the brain

was associated with improved mental functions as measured in the Morris Water Maze test. This spatially oriented learning test measures an animal's ability to develop a strategy to find a hidden platform (acquisition), remember where the platform was located (probe), relearn where the platform was originally placed (relearning), and finally, to develop a strategy to find a hidden platform located in a new position (relocation). The fact that the treated MPS VII mice perform better than the untreated MPS VII mice in every phase of the test strongly suggests that some of the defects in multiple higher mental functions are prevented. Before this study, it was not clear whether prevention of lysosomal storage in neurons of the CNS early in life would result in improved mental functions, or whether the functional deficits were acquired earlier in development and could not be corrected, even if storage were eliminated. Numerous studies in animal models of lysosomal storage diseases have demonstrated either enzyme-positive cells or an actual reduction of lysosomal storage in the CNS after various therapeutic approaches $(3,10,25-29)$. However, no improvements in mental functions were documented in these studies. One study did demonstrate a reduction in the hydrocephalus and a stabilization of intelligence quotients in children with Hurler's disease (MPS I) after allogeneic bone marrow transplantation (30).

Improved performance in the Morris Water Maze test by treated MPS VII mice correlates with a reduction of lysosomal storage in specific neurons of the brain. We have shown here and previously (18-20) that lysosomal storage material is not completely eliminated from the CNS after enzyme replacement therapy. However, lysosomal storage is reduced in neocortical neurons of treated MPS VII mice. The neocortex has been shown to be involved in spatial learning. Rats with cortical lesions have significant deficits that are measurable by this test (31). Therefore, a reduction in lysosomal storage in neurons of the neocortex in treated MPS VII mice is consistent with improvements in spacial learning.

An alternate explanation for the results could be that the shorter latencies observed for treated MPS VII mice in several phases of the Morris Water Maze test are due to a reduction in the physical impairments associated with the disease. However, several lines of evidence argue that the improved performance is due to improved CNS function. First, there were no significant differences in the latencies to find a visible platform among any of the four groups of mice (data not shown). This result suggests that, at this age, untreated MPS VII mice (a) can see the platform; $(b)$ can swim well, and $(c)$ are equally motivated to escape from the water. Second, treated MPS VII mice perform significantly better in the probe phase (which is less sensitive to differences in mobility than are the acquisition, relearning, or relocation phases) than do untreated MPS VII mice. Finally, the correlation between improvements in the Morris Water Maze and a reduction in lysosomal storage in neurons of the neocortex is consistent with studies in rats showing that this structure is involved in spatial learning.

An unexpected finding in this study is that treated normal mice performed poorly in every phase of the Morris Water Maze test. It is not clear why this group of mice would have spacial learning deficits since there are no obvious histologic abnormalities in the brains of these animals. However, these data suggest that excess $\beta$-glucuronidase may exert direct or indirect toxic effects on the CNS. We previously showed that the same dose of enzyme delivered to newborn MPS VII mice 
resulted in $\sim 30 \%$ normal levels of activity in the brain $1 \mathrm{~h}$ after an intravenous injection (17). This additional enzyme in the normal mouse may result in aberrant occupancy and internalization of the mannose-6-phosphate/insulin-like growth factor II (Man-6-P/IGFII) receptor (32), possibly leading to subtle developmental defects and behavioral abnormalities. The recent determination of the $\beta$-glucuronidase crystal structure (33) and delineation of the active site should make it possible to treat normal mice with active and inactive forms of the enzyme to determine if receptor occupancy or enzymatic activity are responsible for these findings. It may also be informative to compare the behavioral performance of transgenic mice overexpressing $\beta$-glucuronidase (34) to that of normal and MPS VII mice. Alternatively, delivery of high doses of exogenous enzyme may have indirect effects on the CNS by altering the normal levels or composition of glucuronides of hormones or other metabolites such as bilirubin (35). Cleavage of glucuronic acid residues from these compounds may alter their biological activity or enhance their toxicity. Since the enzyme injections were started shortly after birth when the blood/brain barrier is incompletely formed (36), the modified compounds may have gained access to the brain and affected normal development of the CNS.

We showed previously that the auditory defects in the MPS VII mouse could be reduced by syngeneic BMT performed at birth (8). It was not clear whether improvements in auditory function could also be achieved in MPS VII mice after enzyme replacement since there are significant differences between the two therapies. Both syngeneic BMT and enzyme replacement performed at birth result in dramatic phenotypic improvements in MPS VII mice. However, enzyme replacement reduced lysosomal storage in neurons of the CNS, but did not prevent accumulation of storage in the cornea (18-20). In contrast, BMT did not significantly reduce storage in neurons of the CNS, but did clear the corneal fibroblasts of storage (9, 10). Here we show that enzyme replacement initiated at birth resulted in dramatic improvements in auditory function. The improvement is of similar magnitude to that observed in MPS VII mice after BMT performed at birth (8). The finding that early initiation of therapy in the MPS VII mouse results in improved hearing is consistent with results showing that children with MPS II have improved hearing after allogeneic BMT (37).

Direct enzyme replacement with recombinant proteins has now been used successfully for multiple lysosomal storage diseases in addition to MPS VII. Lysosomal storage has been reduced in cultured fibroblasts from patients with Hunter syndrome (MPS II), Hurler syndrome (MPS I), and Pompe disease (GSD II) by direct replacement of the deficient lysosomal enzyme (38-40). Intravenous injections of recombinant $\alpha$-L-iduronidase and $N$-acetylgalactosamine-4-sulfatase reduced lysosomal storage material in multiple tissues in a canine model of MPS I and a feline model of Maroteaux-Lamy syndrome (MPS VI), respectively $(41,42)$. Children injected with modified glucocerebrosidase have less severe hepatosplenomegaly and fewer bone and joint abnormalities (43, 44). Although direct enzyme replacement appears to be effective for multiple lysosomal storage diseases, the extreme cost of lifelong treatment may preclude its routine use. However, it may be appropriate in combination with other strategies such as BMT or gene therapy. It appears that some of the beneficial effects of enzyme replacement and BMT are complimentary (20) and a combination of early enzyme replacement followed by BMT or gene therapy directed to the hematopoietic system may have long-term therapeutic effects. An additional form of therapy that may compliment the early beneficial effects of ERT is the use of genetically modified organoids that overproduce lysosomal enzymes (13). Since a combination of therapies may be the most practical long-term approach for this group of diseases, it is crucial to determine if the functional improvements documented here persist when enzyme replacement is augmented with BMT. It is also critical to determine when and if the defects in behavior and hearing return after the treatments are terminated.

\section{Acknowledgments}

We thank Tom Daly and Marie Roberts for critical reading of the manuscript.

This work was partially supported by National Institutes of Health grants DK-50158 to M.S. Sands, DK-41082 to C. Vogler, and GM-34182 to W.S. Sly.

\section{References}

1. Neufeld, E.F., and J. Muenzer. 1989. The mucopolysaccharidoses. In The Metabolic Basis of Inherited Disease. C.R. Scriver, A.L. Beaudet, W. Sly, and D. Valle, editors. McGraw-Hill Inc., New York. 1565-1587.

2. Sly, W.S., B.A. Quinton, W.H. McAllister, and D.L. Rimoin. 1973. $\beta$-Glucuronidase deficiency: report of clinical, radiologic, and biochemical features of a new mucopolysaccharidosis. J. Pediatr. 82:249-257.

3. Birkenmeier, E.H., M.T. Davisson, W.G. Beamer, R.E. Ganschow, C.A. Vogler, B. Gwynn, K.A. Lyford, L.M. Maltais, and C.J. Wawrzyniak. 1989. Murine mucopolysaccharidosis VII. Characterization of a mouse with $\beta$-glucuronidase deficiency. J. Clin. Invest. 83:1258-1266.

4. Sands, M.S., and E.H. Birkenmeier. 1993. A single-base-pair deletion in the $\beta$-glucuronidase gene accounts for the phenotype of murine mucopolysaccharidosis type VII. Proc. Natl. Acad. Sci. USA. 90:6567-6571.

5. Vogler, C., E.H. Birkenmeier, W.S. Sly, B. Levy, C. Pegors, J.W. Kyle, and W.G. Beamer. 1990. A murine model of mucopolysaccharidosis VII. Gross and microscopic findings in $\beta$-glucuronidase-deficient mice. Am. J. Pathol. 136: 207-217.

6. Chang, P.L., D.T. Lambert, and M.A. Pisa. 1993. Behavioral abnormalities in a murine model of a human lysosomal storage disease. Neuro. Rep. 4: 507-510.

7. Bastedo, L., M.S. Sands, D.T. Lambert, M.A. Pisa, E.H. Birkenmeier, and P.L. Chang. 1994. Behavioral consequences of bone marrow transplantation in the treatment of murine mucopolysaccharidosis type VII. J. Clin. Invest. 94:1180-1186.

8. Sands, M.S., L.C. Erway, C. Vogler, W.S. Sly, and E.H. Birkenmeier. 1995. Syngeneic bone marrow transplantation reduces the hearing loss associated with murine mucopolysaccharidosis type VII. Blood. 86:2033-2040.

9. Birkenmeier, E.H., J.E. Barker, C.A. Vogler, J.W. Kyle, W.S. Sly, B. Gwynn, B. Levy, and C. Pegors. 1991. Increased life span and correction of the metabolic defects in murine mucopolysaccharidosis type VII after syngeneic bone marrow transplantation. Blood. 78:3081-3092.

10. Sands, M.S., J.E. Barker, C. Vogler, B. Levy, B. Gwynn, N. Galvin, W.S. Sly, and E.H. Birkenmeier. 1993. Treatment of murine mucopolysaccharidosis type VII by syngeneic bone marrow transplantation in neonates. Lab. Invest. 68:676-686.

11. Snyder, E.Y., R.M. Taylor, and J.H. Wolfe. 1995. Neuronal progenitor cell engraftment corrects lysosomal storage throughout the MPS VII mouse brain. Nature. 374:367-370.

12. Wolfe, J.H., M.S. Sands, J.E. Barker, B. Gwynn, L. Rowe, C.A. Vogler, and E.H. Birkenmeier. 1992. Reversal of pathology in murine mucopolysaccharidosis type VII by somatic cell gene transfer. Nature. 360:749-753.

13. Moullier, P., D. Bohl, J.M. Heard, and O. Danos. 1993. Correction of lysosomal storage in the liver and spleen of MPS VII mice by implantation of genetically modified skin fibroblasts. Nat. Genet. 4:154-159.

14. Marechal, V., N. Naffakh, O. Danos, and J.M. Heard. 1993. Disappearance of lysosomal storage in spleen and liver of mucopolysaccharidosis VII mice after transplantation of genetically modified bone marrow stem cells. Blood. 82:1358-1365.

15. Li, T., and B.L. Davidson. 1995. Phenotypic correction in retinal epithelium in murine mucopolysaccharidosis type VII by adenovirus-mediated gene transfer. Proc. Natl. Acad. Sci. USA. 92:7700-7704.

16. Taylor, R.M., and J.H. Wolfe. 1997. Decreased lysosomal storage in the adult MPS VII mouse brain in the vicinity of grafts of retroviral-corrected fibro- 
blasts secreting high levels of $\beta$-glucuronidase. Nat. Med. 3:771-774.

17. Vogler, C., M.S. Sands, A. Higgins, B. Levy, J. Grubb, E.H. Birkenmeier, and W.S. Sly. 1993. Enzyme replacement with recombinant $\beta$-glucuronidase in the newborn mucopolysaccharidosis type VII mouse. Pediatr. Res. 34:837-840.

18. Sands, M.S., C. Vogler, J.W. Kyle, J.H. Grubb, B. Levy, N. Galvin, W.S. Sly, and E.H. Birkenmeier. 1994. Enzyme replacement therapy for murine mucopolysaccharidosis type VII. J. Clin. Invest. 93:2324-2331.

19. Vogler, C., M.S. Sands, B. Levy, N. Galvin, E.H. Birkenmeier, and W.S. Sly. 1996. Enzyme replacement with recombinant $\beta$-glucuronidase in murine mucopolysaccharidosis type VII: impact of therapy during the first six weeks of life on subsequent lysosomal storage, growth, and survival. Pediatr. Res. 39: $1050-1054$.

20. Sands, M.S., C. Vogler, A. Torrey, B. Levy, B. Gwynn, J. Grubb, W.S. Sly, and E.H. Birkenmeier. 1997. Murine mucopolysaccharidosis type VII. Long-term therapeutic effects of enzyme replacement and enzyme replacement followed by bone marrow transplantation. J. Clin. Invest. 99:1596-1605.

21. Grubb, J.H., J.W. Kyle, L.B. Cody, and W.S. Sly. 1993. Large scale purification of phosphorylated recombinant human $\beta$-glucuronidase from overexpressing mouse L cells. FASEB J. 7(Abstr.):1255.

22. Wolfe, J.H., and M.S. Sands. 1996. Murine mucopolysaccharidosis type VII: a model system for somatic gene therapy of the central nervous system. In Gene Transfer Into Neurones, Towards Gene Therapy of Neurological Disorders. P. Lowenstein and L. Enquist, editors. J. Wiley and Sons, Essex, England. 263-274

23. Morris, R. 1984. Developments of a water-maze procedure for studying spatial learning in the rat. J. Neurosci. Methods. 11:47-60.

24. Berry, C.L., C. Vogler, N.J. Galvin, E.H. Birkenmeier, and W.S. Sly. 1994. Pathology of the ear in murine mucopolysaccharidosis type VII: morphological correlates of hearing loss. Lab. Invest. 71:438-445.

25. Yaeger, A.M., S. Brennan, C. Tiffany, H.W. Moser, and G.W. Santos. 1984. Prolonged survival and remyelination after hematopoietic cell transplantation in the twitcher mouse. Science. 225:1052-1054.

26. Hoogerbrugge, P.M., K. Suzuki, K. Suzuki, B.J.H.M. Poorthuis, T. Kobayashi, G. Wagemaker, and D.W. van Bekkum. 1988. Donor-derived cells in the central nervous system of twitcher mice after bone marrow transplantation. Science. 239:1035-1038.

27. Taylor, R.M., G.J. Stewart, B.R.H. Farrow, and P.J. Healy. 1986. Enzyme replacement in nervous tissue after allogeneic bone marrow transplantation for fucosidosis in dogs. Lancet. 2:772-774.

28. Shull, R.M., N.E. Hastings, R.R. Selcer, J.B. Jones, J.R. Smith, W.C. Cullen, and G. Constantopoulos. 1987. Bone marrow transplantation in canine mucopolysaccharidosis I. Effects within the central nervous system. J. Clin. Invest. 79:435-443.

29. Walkley, S.U., M.A. Thrall, K. Dobrenis, M. Huang, P.A. March, D.A. Siegel, and S. Wurzelmann. 1994. Bone marrow transplantation corrects the enzyme defect in neurons of the central nervous system in a lysosomal storage disease. Proc. Natl. Acad. Sci. USA. 91:2970-2974.

30. Whitley, C.B., K.G. Belani, P.N. Chang, C.G. Summers, B.R. Blazar, M.Y. Tsai, R.E. Latchaw, N.K.C. Ramsay, and J.H. Kersey. 1993. Long-term outcome of Hurler syndrome following bone marrow transplantation. Am. J. Med. Genet. 46:209-218.

31. Kolb, B., R.J. Sutherland, and I.Q. Whishaw. 1983. A comparison of the contributions of the frontal and parietal association cortex to spacial localization in rats. Behav. Neurosci. 97:1-27.

32. Kornfeld, S. 1992. Structure and function of the mannose-6-phosphate insulin like growth factor II receptors. Annu. Rev. Biochem. 61:307-330.

33. Jain, S., W.B. Drendel, Z.W. Chen, F.S. Mathews, W.S. Sly, and J.H Grubb. 1996. Structure of human $\beta$-glucuronidase reveals candidate lysosomal targeting and active-site motifs. Nat. Struct. Biol. 3:375-381.

34. Kyle, J.W., E.H. Birkenmeier, B. Gwynn, C. Vogler, P.C. Hoppe, J.W Hoffmann, and W.S. Sly. 1990. Correction of murine mucopolysaccharidosis type VII by a human $\beta$-glucuronidase transgene. Proc. Nat. Acad. Sci. USA. 87: 3914-3918.

35. Clarke, D.J., and B. Burchell. 1994. The uridine diphosphate glucuronosyltransferase multigene family: function and regulation. In Conjugation-Deconjugation Reactions in Drug Metabolism. F.C. Kauffman, editor. SpringerVerlag, Berlin. 3-43.

36. Lattera, J.J., P.A. Stewart, and G.W. Goldstein. 1992. Development of the blood brain barrier. In Fetal and Neonatal Physiology. A. Polin and W.W. Fox, editors. W.B. Saunders Co., Philadelphia. 1525-1531.

37. Krivit, W., L.A. Lockman, P.A. Watkins, J. Hirsch, and E.G. Shapiro 1995. The future of bone marrow transplantation as a treatment for adrenoleukodystrophy, metachromatic leukodystrophy, globoid cell leukodystrophy, and Hurler syndrome. J. Inherited Metab. Dis. 18:398-412.

38. Bielicki, J., J.J. Hopwood, P.J. Wilson, and D.S. Anson. 1993. Recombinant human iduronate-2-sulphatase: correction of mucopolysaccharidosis type II fibroblasts and characterization of the purified enzyme. Biochem. J. 289:241246

39. Unger, E.G., J. Durrant, D.S. Anson, and J.J. Hopwood. 1994. Recombinant $\alpha$-L-iduronidase: characterization of the purified enzyme and correction of mucopolysaccharidosis type I fibroblasts. Biochem. J. 304:43-49.

40. Fuller, M., A. Van Der Ploeg, A.J.J. Reuser, D.S. Anson, and J.J. Hopwood. 1995. Isolation and characterization of a recombinant, precursor form of lysosomal acid $\beta$-glucosidase. Eur. J. Biochem. 234:903-909.

41. Shull, R.M., E.D. Kakkis, M.F. McEntee, S.A. Kania, A.J. Jonas, and E.F. Neufeld. 1994. Enzyme replacement in a canine model of Hurler syndrome. Proc. Natl. Acad. Sci. USA. 91:12937-12941.

42. Crawley, A.C., D.A. Brooks, V.J. Muller, B.A. Petersen, E.L. Isaac, J Bielicki, B.M. King, C.D. Boulter, A.J. Moore, N.L. Fazzalari, et al. 1996. Enzyme replacement therapy in a feline model of Maroteaux-Lamy Syndrome. $J$. Clin. Invest. 97:1864-1873.

43. Barton, N.W., R.O. Brady, J.M. Dambrosia, A.M. Di Bisceglie, S.H. Dopplet, S.C. Hill, H.J. Mankin, G.J. Murray, R.I. Parker, et al. 1991. Replacement therapy for inherited enzyme deficiency-macrophage-targeted glucocerebrosidase for Gaucher's disease. N. Engl. J. Med. 324:1464-1470.

44. Beutler, E., A. Kay, A. Saven, P. Garver, D. Thurston, A. Dawson, and B. Rosenbloom. 1991. Enzyme replacement therapy for Gaucher's disease. Blood. 78:1183-1189. 